\title{
New Results about Digital Intersections
}

\author{
Isabelle Sivignon $^{1}$, Florent Dupont ${ }^{2}$, and Jean-Marc Chassery ${ }^{1}$ \\ 1 Laboratoire LIS \\ Domaine universitaire Grenoble - BP46 \\ 38402 St Martin d'Hères Cedex, France \\ \{sivignon, chassery\}@lis.inpg.fr \\ 2 Laboratoire LIRIS - Université Claude Bernard Lyon 1 \\ Bâtiment Nautibus - 8, boulevard Niels Bohr \\ 69622 Villeurbanne cedex, France \\ fdupont@liris.cnrs.fr
}

\begin{abstract}
Digital geometry is very different from Euclidean geometry in many ways and the intersection of two digital lines or planes is often used to illustrate those differences. Nevertheless, while digital lines and planes are widely studied in many areas, very few works deal with the intersection of such objects. In this paper, we investigate the geometrical and arithmetical properties of those objects. More precisely, we give some new results about the connectivity, periodicity and minimal parameters of the intersection of two digital lines or planes.
\end{abstract}

Keywords: Digital straight lines and planes, intersection.

\section{Introduction}

Digital straight lines and digital planes properties have been widely studied in many fields like topology, geometry and arithmetics. Topologically, those object are well defined according to the digitization scheme employed. On the geometrical ground, connectivity features have been determined and a characterization using convex hull properties [1] has been proposed. Finally, an arithmetical definition [2]3] provides a general model to handle all the definitions proposed so far.

Those properties led to many recognition algorithms. Geometric algorithms [4] decide whether a set of pixels/voxels is a digital line/plane or not, and arithmetical algorithms [5] moreover return, for a given digitization scheme, the parameters of the Euclidean lines/planes the digitization of which contains the set of pixels/voxels.

Discrete geometry is different from Euclidean geometry in many ways, but the differences between the intersection of two Euclidean lines and two digital lines is often used to illustrate this difference. Indeed, while the intersection of two Euclidean lines is a Euclidean point, the intersection of two digital lines can be a discrete point, a set of discrete points or even empty on rectangular grids.

However, only a few works deal with the properties of digital lines or planes intersections. Nevertheless, a good knowledge of those objects is useful, for in- 


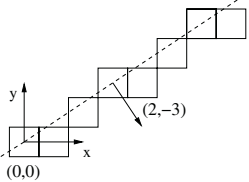

(a)

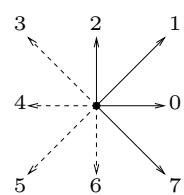

(b)

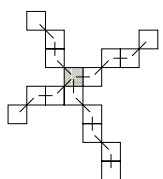

(c)

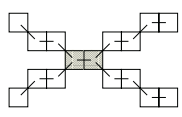

(d)

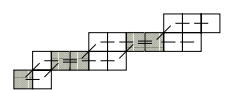

(e)

Fig. 1. (a) The digital naive line $(2,-3,0)$; (b)Freeman code; (c) Two naive lines with no common direction; (d) Two naive lines with one common direction; (e) Two naive lines in the same octant.

stance during the polygonalization process of a discrete curve or a discrete surface. Indeed, this process implies the definition of edges and vertices that are to be found in the intersection of digital lines in the case of polygonal curves or digital planes in the case of digital surfaces.

In [6], using the arithmetical definition of a discrete line/plane, Debled et al. present a definition of the set of intersection pixels/voxels of two digital lines/planes using an unimodular matrix. This definition enables the design of an efficient algorithm to determine all the pixels/voxels of an intersection given the parameters of the two lines/planes. However, no results are given about the topology and arithmetics of this intersection.

In this paper, we present new results about digital lines and digital planes intersection. We focus our study on two properties that describe both topology and arithmetics: connectivity and minimal parameters. The first part deals with the intersection of two digital lines. We present a criterion to analyze the connectivity of the intersection of any two digital lines, thus completing the results presented in [2] for lines with slopes between 0 and 1 . Then, we propose a study about the minimal arithmetic parameters of digital lines intersection and give a result allowing to design an efficient algorithm to find those parameters. The second part deals with digital planes intersection: after some results about connectivity characteristics, we prove that the intersection is periodic and give the minimal period. Finally, we define and determine the minimal parameters of the intersection of two digital planes.

\section{Digital Lines Intersection}

In this section, we focus on the properties of digital lines intersections. A digital naive line of parameters $(a, b, \mu)$ is the set of integer points $\{(x, y)\}$ fulfilling the conditions $0 \leq a x+b y+\mu<\max (|a|,|b|)$. An illustration is proposed in Figure 1(a). Let us consider two digital naive lines denoted $L_{1}$ and $L_{2} . L_{1} \cap L_{2}$ is a set of pixels the connectivity of which depends on the parameters of the two digital lines. 


\subsection{Connectivity}

In 1991, J.-P. Reveillès 2 proposed a criterion to determine whether the intersection of two digital naive lines with slopes between 0 and 1 is connected or not. Nevertheless, he does not give any information about the intersection of any two digital naive lines. We propose here such a criterion using the Freeman code depicted on Figure 1(b). These directions define 8 octants but only 4 remain if we consider symmetries around the central point. For instance, the octant $\{4,5\}$ is equivalent to the octant $\{0,1\}$. A classical result is that the Freeman code of any digital naive line is composed of at most two consecutive different directions, which means that one digital line belongs to one octant.

Proposition 1. Let $L_{1}$ and $L_{2}$ be two digital naive lines. Then:

- if they belong to the same octant, their intersection may be not connected, and [2] gives a criterion to analyze exactly the connectivity;

- if they belong to two neighbors octants, their intersection is either empty or connected;

- otherwise, their intersection is either empty or reduced to a unique pixel.

In the following we denote $F_{1}$ (resp. $F_{2}$ ) the set of directions composing the Freeman code of $L_{1}$ (resp. $L_{2}$ ). An illustration is given Figure 1.

Proof. Let $L_{1}$ and $L_{2}$ be two digital naive lines. If $L_{1}$ and $L_{2}$ belong to the same octant, $\left|F_{1} \cap F_{2}\right|=2$. If they belong to neighbor octants, $\left|F_{1} \cap F_{2}\right|=1$. Otherwise $\left|F_{1} \cap F_{2}\right|=0$. Let us give a classification of the pixels of $L_{1}$ and $L_{2}$. We denote $p_{1, k}=p_{2, k}=p_{k}$ the pixel of $L_{1} \cap L_{2}$ with minimal x-coordinate and maximal $\mathrm{y}$-coordinate, if there exist one. Then, $p_{1, k+1}$ (resp. $p_{2, k+1}$ ) is the successor of $p_{k}$ along $L_{1}$ with increasing x-coordinate (resp. $\left.L_{2}\right)$.

- if $\left|F_{1} \cap F_{2}\right|=0$ (Figure 1(c)), then $p_{1, k+1} \neq p_{2, k+1}$ as they are the successors of the same point using two different directions. Suppose that $L_{1}$ is composed of 0 and 1 freeman codes, and that $L_{2}$ is composed of 2 and 7 . The other cases are symmetrical. Then, let us consider a pixel $p_{1}\left(x_{p}, y_{p_{1}}\right) \in L_{1}$ with $x_{p}$ greater that the $x$-coordinate $x_{k}$ of $p_{k}$, and $p_{2}\left(x_{p}, y_{p_{2}}\right) \in L_{2}$. Then, $y_{k} \leq y_{p_{1}}$ and $y_{p_{2}} \leq y_{k}-\left(x_{p}-x_{k}\right)$, with $x_{p}>=x_{k}$. Hence, the two lines do not have any common point after $p_{k}$.

- if $\left|F_{1} \cap F_{2}\right|=1$ (Figure 1(d)) then let us denote $\alpha_{1 i}$ (resp. $\alpha_{2 i}$ ) the direction used from $p_{1, i}$ to $p_{1, i+1}$ (resp. $p_{2, i}$ to $p_{2, i+1}$ ). Hence, while $\alpha_{1 i}=\alpha_{2 i}, i \geq k$, $p_{1, i+1}=p_{2, i+1}$. Both pixels $p_{1, i}$ and $p_{1, i+1}$ belong to the intersection and are 8-connected. Unless the two lines are confounded, there exist $j$ such that $\alpha_{1 j} \neq \alpha_{2 j}$. Hence, $p_{1, j+1} \neq p_{2, j+1}$. Suppose that $L_{1}$ is composed of 0 and 1 freeman codes, and that $L_{2}$ is composed of 1 and 2 . The other cases are symmetrical. Then, let us consider a pixel $p_{1}\left(x_{p}, y_{p_{1}}\right) \in L_{1}$ with $x_{p}$ greater that the $x$-coordinate $x_{j}$ of $p_{1, j}=p_{2, j}$, and $p_{2}\left(x_{p}, y_{p_{2}}\right) \in L_{2}$. Then, $y_{p_{1}} \leq y_{j}+\left(x_{p}-x_{j}-1\right)$ and $y_{p_{2}} \geq y_{j}+\left(x_{p}-x_{j}\right)$. Hence, the two lines do not have any common point after $p_{1, j}$.

- if $\left|F_{1} \cap F_{2}\right|=2$ (Figure प(e)), we refer to [2] to analyze the connectivity. 


\subsection{Minimal Parameters}

The intersection of two digital lines is a set of collinear discrete points. To characterize this set of points, it is interesting to know the straight lines which digitization contains all the intersection pixels. Obviously, the two lines we are studying are solutions.

Consider a straight line $y=\alpha_{0} x+\beta_{0}, 0 \leq \alpha_{0}, \beta_{0} \leq 1$, thus in the octant $\{0,1\}$. Its digitization with the Object Boundary Quantization is the set of discrete points lying on or just under the line. Given a set of discrete points $\mathcal{P}$, we call preimage and denote $D(\mathcal{P})$ the set of straight lines $(\alpha, \beta): y=\alpha x+\beta$, the $O B Q$ digitization of which contains the discrete points $\mathcal{P}$.

Definition 1. Let $\mathcal{P}$ be a set of discrete points and $D(\mathcal{P})$ its preimage. The minimal parameters of $\mathcal{P}$ are the values $\left(\frac{a}{b}, \frac{\mu}{b}\right) \in D(\mathcal{P})$ such that $b$ and $\mu$ are minimal.

In the following, we show how to find the minimal parameters of the intersection of any two digital naive lines using two different methods and emphasizing the links between them.

Preimage Study. First of all, we show how to find the directional vector of the minimal parameters studying the structure of the intersection preimage.

To study the intersection of any two digital lines, we need to work in the same straight line parameter space for any slope, greater or smaller than 1 . In [7, Veelaert shows that the transformation between the space where $a \geq b$ into the space where $a \leq b$ can be done with a central symmetry in a 3D space. Thus, we can work in the straight line parameter space where a point $(\alpha, \beta)$ represents the line $y=\alpha x+\beta$, for all $\alpha$ and $\beta$. In this space, the preimage of a digital straight line of slope $\frac{a}{b}$ with $a \leq b$ and no remainder is the segment $\left[\left(\frac{a}{b}, 0\right),\left(\frac{a}{b}, \frac{1}{b}\right)\right]$, and the preimage of a digital straight line of slope $\frac{a}{b}$ with $a \geq b$ and no remainder is the segment $\left[\left(\frac{a}{b}, 0\right),\left(\frac{a}{b},-\frac{1}{b}\right)\right]$. For instance, the preimage of the line of slope 1 is the segment $[(1,-1),(1,1)]$ in the parameter space.

We consider two digital naive lines $L_{1}$ and $L_{2}$ with slopes $\frac{a}{b}$ and $\frac{c}{d}$ and no remainder, and their intersection $I=L_{1} \cap L_{2}$. Without loss of generality, we assume that $\frac{a}{b}<\frac{c}{d}$. We denote $D\left(L_{1}\right)$ (resp. $D\left(L_{2}\right)$ ) the preimage of $L_{1}$ (resp. $L_{2}$ ). The preimage $D(I)$ of $L_{1} \cap L_{2}$ is a convex polygon including $D\left(L_{1}\right)$ and $D\left(L_{2}\right)$, and its convexity implies that it includes the segment $\left[\left(\frac{a}{b}, 0\right),\left(\frac{c}{d}, 0\right)\right]$ (see Figure 2 for illustrations). Moreover, as $I$ contains all the discrete points belonging simultaneously to $L_{1}$ and $L_{2}$, adding one more pixel of $L_{1}$ or $L_{2}$ to $I$ cuts $D(I)$ into two parts, one including $D\left(L_{1}\right)$ and the other including $D\left(L_{2}\right)$.

Theorem 1. The minimal directional vector of the intersection of two lines of slopes $\frac{a}{b}$ and $\frac{c}{d}, \frac{a}{b}<\frac{c}{d}$ is given by the rational fraction $\frac{u}{v}$ lying between $\frac{a}{b}$ and $\frac{c}{d}$ with minimal denominator $v$.

Proof. Consider the set of discrete points belonging to $L_{1}$ and $L_{2}, I=L_{1} \cap L_{2}$ and call $D(I)$ its preimage. We divide the proof of the theorem into 3 cases that are depicted in Figure 2 


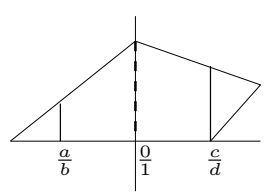

(a)

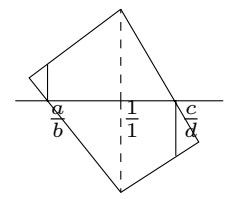

(b)

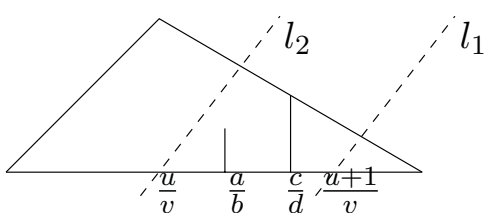

(c)

Fig. 2. Illustration of the three cases of Theorem 1

- Assume that $\frac{a}{b} \leq 0$ and $\frac{c}{d} \geq 0$. Then, the fraction $\frac{0}{1}$ lies between $\frac{a}{b}$ and $\frac{c}{d}$. Consequently, the line with slope $\frac{0}{1}$ is a solution, and obviously the solution with minimal denominator. (cf. Figure $2 \mathrm{a}$ )

- Assume that $\frac{a}{b} \leq 1$ and $\frac{c}{d} \geq 1$. Then, the fraction $\frac{1}{1}$ lies between $\frac{a}{b}$ and $\frac{c}{d}$, and from what we said before, we deduce that the line with slope $\frac{1}{1}$ is a solution, and by the way the one with minimal denominator.(cf. Figure 2 $\mathbf{b}$ )

- Assume that $0 \leq \frac{a}{b}<\frac{c}{d} \leq 1$. We know that any fraction between $\frac{a}{b}$ and $\frac{c}{d}$ is a solution. By the way, the fraction with minimal denominator lying between $\frac{a}{b}$ and $\frac{c}{d}$ is a solution. We show that there does not exist a solution fraction with a smaller denominator outside the segment defined by $\frac{a}{b}$ and $\frac{c}{d}$. Suppose that there exist such a fraction denoted $\frac{u}{v}$. Then, $v<b$ and $v<d$. Suppose that $\frac{u}{v}<\frac{a}{b}$ and that $\left|\frac{a}{b}-\frac{u}{v}\right|$ is minimal for the set of irreducible fractions smaller than $\frac{a}{b}$ with denominator $v$. The case $\frac{u}{v}>\frac{c}{d}$ is symmetrical. Consider the discrete point $p(-v,-u-1)$. Adding this point to $L_{1} \cap L_{2}$ implies two new half-spaces constraints given by $0 \leq-\alpha v+u+1+\beta<1$ in the straight lines parameter space. This strip is delimited by two lines $l_{1}:-\alpha v+u+1+\beta=0$ and $l_{2}:-\alpha v+u+1+\beta=1$. $l_{1}$ cuts the x-coordinate axis for $x=\frac{u+1}{v}$ and $l_{2}$ for $x=\frac{u}{v}$ (see Figure 2x).Thus, since $v$ is smaller than any denominators of the fractions lying between $\frac{a}{b}$ and $\frac{c}{d}, \frac{u+1}{v}$ is either greater than $\frac{c}{d}$ or smaller than $\frac{a}{b}$. But since we assume that $\frac{u}{v}$ was the closest fraction with denominator $v$ smaller than $\frac{a}{b}$, we get that $\frac{u}{v}<\frac{a}{b}<\frac{c}{d}<\frac{u+1}{v}$. Finally, $D(I \cup p)$ includes at the same time $D\left(L_{1}\right)$ and $D\left(L_{2}\right)$, which leads to the contradiction.

All the remaining cases can be treated as one of those three.

Geometrical Method. The preimage study gives us the value of the minimal directional vector of the intersection of two digital lines. We propose here a geometrical point of view that leads to an algorithm to find both the minimal directional vector and the corresponding remainder.

To do so, let us introduce a structure called Stern-Brocot tree (see [8] for a complete definition or [9] for a more informal approach) which contains all the positive irreducible rational fractions. An illustration of this tree is proposed in figure 3(a). The idea under its construction is to begin with the two fractions $\frac{0}{1}$ and $\frac{1}{0}$ and to repeat the insertion of the median of these two fractions as follows: insert the median $\frac{m+m^{\prime}}{n+n^{\prime}}$ between $\frac{m}{n}$ and $\frac{m^{\prime}}{n^{\prime}}$. Many works deal with the 


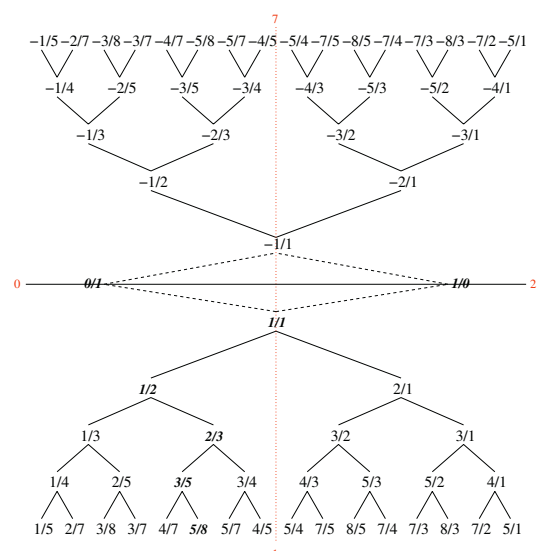

(a)

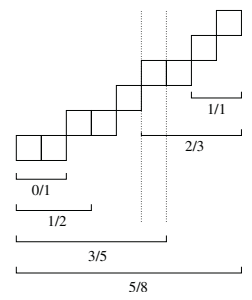

(b)

Fig. 3. (a) Stern-Brocot tree: positive and negative irreducible rational fractions. (b) Decomposition of one period of the digital line of slope $\frac{5}{8}$ : for each fraction of the path in the Stern-Brocot tree, the corresponding subset of pixels of the line.

relations between irreducible rational fractions and digital lines (see [10]1] for a characterization with Farey series, and [12] for a link with decomposition into continuous fractions), but in [5], Debled first introduced the link between this tree and digital lines. She noticed that recognizing a piece of digital line is like going down the Stern-Brocot tree up to the directional vector of the line. In the following, we call Stern-Brocot tree root the two fractions $\frac{0}{1}$ and $\frac{1}{0}$.

Theorem 2. Let $L$ be a digital line of slope $\frac{a}{b}$, and $S\left(\frac{a}{b}\right)$ be the path going from the Stern-Brocot tree root to the fraction $\frac{a}{b}$.

Then, for each fraction $\frac{a_{i}}{b_{i}}$ lying on $S\left(\frac{a}{b}\right)$, there exist a subset of $b_{i}+1$ pixels of $L$ having a minimal directional vector $\frac{a_{i}}{b_{i}}$.

Moreover, for any other fraction, there does not exist such a subset of $L$.

This theorem means that the path leading to the fraction $\frac{a}{b}$ represents all the patterns of length smaller than $b$ included in $L$. If $b=0$ for a given digital line, then we consider the fraction $\frac{b}{a}$ and the same results hold.

Before the proof of this theorem, let us give a few lemmas. The proof of lemma 1 was given by Dorst and Duin in 13.

Lemma 1. Let $L_{1}$ and $L_{2}$ be two digital naive lines of slope $\frac{u_{1}}{v_{1}}$ and $\frac{u_{2}}{v_{2}}$ such that $u_{2} v_{1}-u_{1} v_{2}=1$. Let $C_{1}$ (resp. $C_{2}$ ) be the Freeman code associated to a period of $L_{1}$ (resp. $L_{2}$ ) of length $v_{1}+1$ (resp. $\left.v_{2}+1\right)$. Then, the Freeman code associated to a period of the digital naive line of slope $\frac{u_{1}+u_{2}}{v_{1}+v_{2}}$ is $C_{1} C_{2}$ of length $v_{1}+v_{2}+1$.

An illustration of this lemma is given in Figure 3(b). 
We call mothers of a fraction $\frac{u}{v}$ the two fractions $\frac{u_{1}}{v_{1}}$ and $\frac{u_{2}}{v_{2}}$ such that $\frac{u_{1}+u_{2}}{v_{1}+v_{2}}=$ $\frac{u}{v}$. Hence, we have the following result:

Lemma 2. Let $\frac{a}{b}$ an irreducible rational fraction and $S\left(\frac{a}{b}\right)$ its related path. Then, the mothers of $\frac{a}{b}$ lie on $S\left(\frac{a}{b}\right)$. Moreover, if we denote $A\left(\frac{a}{b}\right)$ the set of ancestors of $\frac{a}{b}$ according to the definition of mothers, we have $S\left(\frac{a}{b}\right)=A\left(\frac{a}{b}\right)$.

This lemma is directly derived from the definition and construction of the Stern-Brocot tree.

Proof (Theorem 2). Let $\frac{a}{b}$ an irreducible rational fraction and $S\left(\frac{a}{b}\right)$ its related path. Let $\frac{u}{v} \in S\left(\frac{a}{b}\right)$ another rational fraction. Two possibilities:

- if $\frac{u}{v}$ is one of $\frac{a}{b}$ mothers, then we derive the result from lemma 1.

- otherwise, according to lemma 2, $\frac{u}{v}$ is one of $\frac{a}{b}$ ancestors, and the result is obtained by induction.

$\frac{a}{b}$ ancestors represent all the connected subsets of discrete points that appear in the digital line of slope $\frac{a}{b}$. As $S\left(\frac{a}{b}\right)=A\left(\frac{a}{b}\right)$, there is no fraction outside the path corresponding to a connected pattern of the digital line of slope $\frac{a}{b}$.

Hence, each node of the tree matches with a pattern. Since the intersection of two digital lines is composed of patterns appearing in the two lines, we just have to look for the closest common ancestor of the two corresponding fractions to find the minimal parameters of the intersection,.

Theorem 3. Let $L_{1}$ and $L_{2}$ be two digital lines of slopes $\frac{a_{1}}{b_{1}}$ and $\frac{a_{2}}{b_{2}}$. Then, the minimal parameters of $L_{1} \cap L_{2}$ are given by $\frac{a_{1}}{b_{1}}$ and $\frac{a_{2}}{b_{2}}$ closest common ancestor in the Stern-Brocot tree.

If the two digital lines studied are such that $b_{1}=0$ and $a_{2}=0$, then the corresponding nodes are the root of the Stern-Brocot tree, and the minimal parameters are any of the two fractions of the root.

Originally, the Stern-Brocot tree defines only the positive irreducible rational fractions. In order to study the intersection of any two digital lines, we generalize this tree adding its negative symmetrical as shown on Figure [3(a).

It is easy to see with the preimage study or the geometrical method that the directional vector found for two digital lines with no remainder is also solution for any remainder. Nevertheless, if the cardinal of the intersection is smaller than the length of the common pattern described by the directional vector found, there exist smaller parameters. In that case, the minimal directional vector can be found among the common ancestors of the two fractions in the Stern-Brocot tree, looking for the one with the smallest denominator greater than or equal to the intersection cardinal minus 1.

Theorems 1 and 3 are equivalent as looking for the closest common ancestor of two fractions is the Stern-Brocot tree is like looking for the fraction with minimal denominator lying between those two fractions. Nevertheless, this geometrical point of view is useful to design an efficient algorithm to determine the 


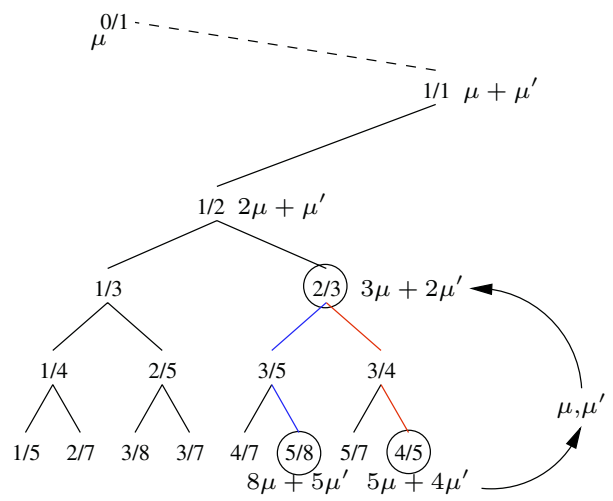

Fig. 4. Remainder calculation for the digital lines $\left(4,-5, \mu_{1}\right)$ et $\left(5,-8, \mu_{2}\right)$.

minimal directional vector. Moreover, we show that this method enables to find the minimal remainder associated to this minimal directional vector.

Let us define the following labelling $\mathcal{L}$ of the Stern-Brocot tree nodes:

$-\mathcal{L}\left(\frac{0}{1}\right)=\mu$ and $\mathcal{L}\left(\frac{1}{0}\right)=\mu^{\prime}$

- let $\frac{a}{b}$ be a node and $\frac{u_{1}}{v_{1}}$ and $\frac{u_{2}}{v_{2}}$ its mothers: then $\mathcal{L}\left(\frac{a}{b}\right)=\mathcal{L}\left(\frac{u_{1}}{v_{1}}\right)+\mathcal{L}\left(\frac{u_{2}}{v_{2}}\right)$.

Finally, $\mathcal{L}\left(\frac{a}{b}\right)=b \mu+a \mu^{\prime}$. Each node label thus depends on only two variables. Now let us consider the intersection of two digital lines $L_{1}\left(a,-b, \mu_{1}\right)$ and $L_{2}\left(c,-d, \mu_{2}\right)$. Mapping the remainder values with the corresponding nodes labels, we get the following system:

$$
\left\{\begin{array}{l}
b \mu+a \mu^{\prime}=\mu_{1} \\
d \mu+c \mu^{\prime}=\mu_{2}
\end{array}\right.
$$

Hence, we can deduce the values of $\mu$ and $\mu^{\prime}$, and injecting those values in the label of the node corresponding to the intersection parameters, we get the remainder of the intersection. Figure 4 illustrates this with an example.

\section{Digital Planes Intersection}

In this part, we extend the properties found on digital lines intersection for digital planes intersection and present some properties peculiar to planes. The grid considered is a square grid with a $26-6$ connectivity.

\subsection{Periodicity}

Proposition 2. Let $P_{1}(a, b, c, \mu)$ and $P_{2}(d, e, f, \nu)$ be two digital planes. Let $v\left(v_{1}, v_{2}, v_{3}\right)^{T}$ be the cross product of $(a, b, c)^{T}$ and $(d, e, f)^{T}$. Let $g=\operatorname{gcd}\left(v_{1}, v_{2}, v_{3}\right)$ and $v^{\prime}=\frac{1}{g} v$. Then $P_{1} \cap P_{2}$ is periodic of period $v^{\prime}$. 
Proof. Let us denote $r_{1}(x, y, z)=a x+b y+c y+\mu$ and $r_{2}(x, y, z)=d x+e y+f y+\nu$ the remainder function of the two planes. Let $M(x, y, z) \in P_{1} \cap P_{2}$. Then $M+t v^{\prime}$ is not an integer point if $t$ is not integer. We show that $M+v^{\prime}$ belongs to $P_{1} \cap P_{2}$ and that $r_{1}\left(M+v^{\prime}\right)=r_{1}(M)$ and $r_{2}\left(M+v^{\prime}\right)=r_{2}(M)$ :

$r_{1}\left(M+v^{\prime}\right)=a x+b y+c z+\mu+\frac{1}{g}(a b f-a c e+b d c-a b f+a c e-b c d)=r_{1}(M)$

The same calculation can be done with the $r_{2}$ function and this achieves that $P_{1} \cap P_{2}$ is periodic of period $v^{\prime}$.

\subsection{Minimal Parameters}

In this part, we focus on the minimal parameters of the intersection of two digital planes. To work in the same parameter space for any parameters, we use the same trick as the one proposed by Veelaert [7] for lines, presented in section 2.2. Hence, we work in the parameter space where a point $\left(\alpha_{0}, \beta_{0}, \gamma_{0}\right)$ stands for the plane $\alpha_{0} x+\beta_{0} y+z+\gamma_{0}=0$ in the Cartesian space for any value of $\alpha_{0}, \beta_{0}$ and $\gamma_{0}$.

Given two digital planes $P_{1}$ and $P_{2}$, we look for the plane parameters $(u, v, w$, $\mu$ ) with minimal $w$ and $\mu$ the $O B Q$ digitization of which contains all the voxels of $P_{1} \cap P_{2}$.

In the following, we consider digital naive planes with no remainder: digital naive planes are the thinnest 18 -connected digital planes without 6 -connected holes. First of all, Proposition 3 gives a description of the intersection preimage.

Proposition 3. Let $P_{1}(a, b, c, 0)$ and $P_{2}(d, e, f, 0)$ be two digital naive planes. We denote $I=P_{1} \cap P_{2}$. Then, $D(I)$ is a polygon included in the plane perpendicular to $\gamma=0$ and containing the points $\left(\frac{a}{c}, \frac{b}{c}, 0\right)$ and $\left(\frac{d}{f}, \frac{e}{f}, 0\right)$.

Proof. Since the two planes have no remainder, the point $(0,0,0)$ is a lower leaning point of the two digital planes. As $I$ is periodic of period $v$ (Theorem 2), for all integer t, the point $t v$ belongs to $P_{1} \cap P_{2}$ and is a lower leaning point of the two digital planes. In the dual space, the point $t v$ corresponds to the two constraints $0 \leq \alpha t v_{1}+\beta t v_{2}+t v_{3}+\gamma<1$. Since $t v$ is a lower leaning point for the two digital planes, the constraint $\alpha t v_{1}+\beta t v_{2}+t v_{3}+\gamma=0$ goes through the two points $\left(\frac{a}{c}, \frac{b}{c}, 0\right)$ and $\left(\frac{d}{f}, \frac{e}{f}, 0\right)$. Hence, for all $t, D(I)$ is constrained by the plane $\alpha t v_{1}+\beta t v_{2}+t v_{3}+\gamma=0$, equivalent to $\alpha v_{1}+\beta v_{2}+v_{3}+\frac{1}{t} \gamma=0$ for $t \neq 0$. When $t$ goes to $+\infty$, the normal vector of this plane converge to the value $\left(v_{1}, v_{2}, 0\right)$ with positive values of $t$ and with negative values of $t$ when $t$ goes to $-\infty$. Then, for infinite planes, $D(I)$ is reduced to a polygon included in the plane with normal vector $\left(v_{1}, v_{2}, 0\right)$ which contains the two points $\left(\frac{a}{c}, \frac{b}{c}, 0\right)$ and $\left(\frac{d}{f}, \frac{e}{f}, 0\right)$.

An example of an intersection preimage is given Figure 5

This description enables to characterize the minimal parameters of $I$ : 


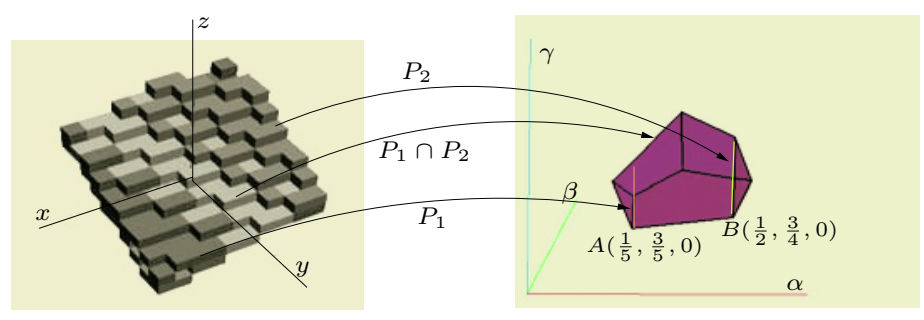

Fig. 5. Preimage of the intersection of the digital naive planes $P_{1}(1,3,5,0)$ and $P_{2}(2,3,4,0)$.

Theorem 4. Let $P_{1}(a, b, c, 0)$ and $P_{2}(d, e, f, 0)$ be two digital naive planes. We denote $A\left(\frac{a}{c}, \frac{b}{c}, 0\right)$ and $B\left(\frac{d}{f}, \frac{e}{f}, 0\right)$ the corresponding points in the parameter space, and $I=P_{1} \cap P_{2}$. Then, the minimal normal vector of $I$ is given by the point $\left(\frac{u}{w}, \frac{v}{w}, 0\right)$ on $[A B]$ with minimal $w$.

Proof. Without loss of generality, we suppose that $\frac{a}{c} \leq \frac{d}{f}$. To prove this theorem, we use the results obtained for digital lines using a digital plane decomposition into digital lines presented in [14. Indeed, we can decompose any digital plane $P(a, b, c, \mu)$ into digital 3D lines: for instance, a decomposition along the $y$ axis gives the set of lines $S_{y j}(P)=\left\{\left(x_{0}, y_{0}, z_{0}\right) \in P \mid y_{0}=j\right\}, \forall j \in \mathbb{Z}$. For two out of these three possible decompositions, those lines are naive lines, and for the third one, they are thicker than naive lines.

Since $I$ is a piece of naive plane, we can use this decomposition. Consider the decomposition of $I$ along the $y$ axis. We denote $S_{y j}(I)$ the $3 \mathrm{D}$ digital lines of this decomposition. Then we have $D(I)=\bigcap_{j} D\left(S_{y j}(I)\right)$. Moreover, $S_{y j}(I)=$ $S_{y j}\left(P_{1} \cap P_{2}\right)=S_{y j}\left(P_{1}\right) \cap S_{y j}\left(P_{2}\right)$ as $S_{y j}(I)$ is the set of pixels of $P_{1} \cap P_{2}$ the $y$-coordinate of which is $j$.

Let us consider the set $S_{y 0}(I)=S_{y 0}\left(P_{1}\right) \cap S_{y 0}\left(P_{2}\right)$. Then, we get two cases:

- if $S_{y 0}\left(P_{1}\right)$ and $S_{y 0}\left(P_{2}\right)$ are naive lines, we denote them $N_{3 D, 1}(a, c, 0)$ and $N_{3 D, 2}(d, f, 0)$. Then, $S_{y 0}(I)=N_{3 D, 1} \cap N_{3 D, 2}$.

- otherwise, $S_{y 0}\left(P_{1}\right)$ or $S_{y 0}\left(P_{2}\right)$ is thicker than a naive line but contains the naive line of the previous case. Thus we have $S_{y 0}(I) \supset N_{3 D, 1} \cap N_{3 D, 2}$.

If we consider the preimages of those sets, we then get the following property: $D\left(S_{y 0}(I)\right) \subseteq D\left(N_{3 D, 1} \cap N_{3 D, 2}\right)$.

$N_{3 D, 1} \cap N_{3 D, 2}$ is a piece of $3 \mathrm{D}$ naive line and its preimage is a prism such that the basis in the plane $\beta=0$ is the preimage of the intersection of the two $2 \mathrm{D}$ naive lines $N_{2 D, 1}(a, c, 0)$ and $N_{2 D, 2}(d, f, 0)$ and such that the directional vector is $(1,0,0)^{T}$.

Let $p\left(\frac{u}{w}, \frac{v}{w}, \frac{k}{w}\right)$ be a point of $D(I)$ as illustrated on Figure 6. Then $p \in$ $D\left(S_{y 0}(I)\right)$ and thus $p \in D\left(N_{3 D, 1} \cap N_{3 D, 2}\right)$. The projection of $p$ along the prism previously described onto the plane $\beta=0$ is the point $\operatorname{proj}(p)\left(\frac{u}{w}, 0,0\right)$. $\operatorname{proj}(p) \in D\left(N_{2 D, 1} \cap N_{2 D, 2}\right)$ and according to the results about the preimage of the intersection of two digital 2D naive lines, if $w<c$ and $w<f$, then 


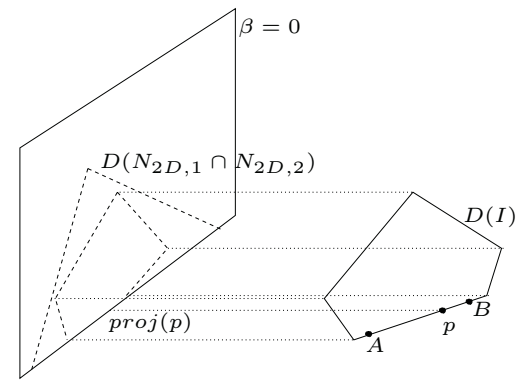

Fig. 6. Illustration of the proof of Theorem 4

$\frac{a}{c} \leq \frac{u}{w} \leq \frac{d}{f}$. If $\frac{a}{c}=\frac{d}{f}$, then $\frac{b}{c} \neq \frac{e}{f}$ and the same argument can be applied using a decomposition along the $x$ axis. Otherwise, finally, we derive that, if $w<c$ and $w<f$, thus $p$ belongs to $[A B]$ from the structure of $D(I)$ presented in Proposition 3. This shows that the minimal parameters are to be found on $[A B]$.

\section{Conclusion}

In this paper, we present new results about the intersection of two digital lines or two digital planes. We give criteria to analyze its connectivity and propose a characterization of the minimal parameters of a given intersection in function of the parameters of the two lines/planes.

Although the properties are enounced and proved for digital naive lines and planes, those results are also true or can be easily transposed for standard objects. For instance, the connectivity results for lines intersections can be adapted transforming any diagonal moving into an horizontal and a vertical one. Moreover, all the results about minimal parameters are based on the intersection preimage features, which depend on the lines or planes preimage shape. But the preimage of a standard line or plane is a translated copy of the preimage of the naive line or plane having the same parameters.

Those properties can be used for instance in the polygonalization process for digital curves and digital surfaces to define edges and vertices and a study of the intersection of two 3D digital lines would be interesting for that problem.

\section{References}

1. Kim, C.E.: Three-dimensional digital planes. IEEE Trans. on Pattern Analysis and Machine Intelligence 6 (1984) 639-645

2. Réveillès, J.P.: Géométrie discrète, calcul en nombres entiers et algorithmique. PhD thesis, Université Louis Pasteur, Strasbourg, France (1991)

3. Andrès, E., Acharya, R., Sibata, C.: Discrete analytical hyperplanes. Graphical Models and Image Processing 59 (1997) 302-309 
4. Kim, C.E., Stojmenovic̀, I.: On the recognition of digital planes in three-dimensional space. Pattern Recognition Letters 12 (1991) 665-669

5. Debled-Rennesson, I.: Etude et reconnaissance des droites et plans discrets. PhD thesis, Université Louis Pasteur, Strasbourg, France (1995)

6. Debled, I., Reveillès, J.P.: A new approach to digital planes. In: Spie's Internat. Symposium on Photonics and Industrial Applications - Technical conference vision geometry 3. (1994) Boston.

7. Veelaert, P.: Geometric constructions in the digital plane. Journal of Mathematical Imaging and Vision 11 (1999) 99-118

8. Hardy, G.H., Wright, E.M.: An introduction to the Theory of Numbers. Oxford Society (1989)

9. Hayes, B.: On the teeth of wheels. In: Computing Science. Volume 88-4., American Scientist (2000) 296-300

10. McIlroy, M.D.: A note on discrete representation of lines. AT\&T Technical Journal 64 (1985) 481-490

11. Dorst, L., Smeulders, A.N.M.: Discrete representation of straight lines. IEEE Trans. on Pattern Analysis and Machine Intelligence 6 (1984) 450-463

12. Yaacoub, J.: Enveloppes convexes de réseaux et applications au traitement d'images. PhD thesis, Université Louis Pasteur, Strasbourg, France (1997)

13. Dorst, L., Duin, R.P.W.: Spirograph theory: A framework for calculations on digitized straight lines. IEEE Trans. on Pattern Anal. and Mach. Intell. 6-5 (1984) 632-639

14. Coeurjolly, D., Sivignon, I., Dupont, F., Feschet, F., Chassery, J.M.: Digital plane preimage structure. In Del Lungo, A., Di Gesù, V., Kuba, A., eds.: Electronic Notes in Discrete Mathematics, IWCIA'03. Volume 12., Elsevier Science Publishers (2003) 